\title{
Quantum entanglement produced in the formation of a black hole
}

\author{
Eduardo Martín-Martínez, ${ }^{1}$ Luis J. Garay, ${ }^{2,3}$ and Juan León ${ }^{1}$ \\ ${ }^{1}$ Instituto de Física Fundamental, CSIC, Serrano 113-B, 28006 Madrid, Spain \\ ${ }^{2}$ Departamento de Física Teórica II, Universidad Complutense de Madrid, 28040 Madrid, Spain \\ ${ }^{3}$ Instituto de Estructura de la Materia, CSIC, Serrano 121, 28006 Madrid, Spain
}

(Received 16 July 2010; published 22 September 2010)

\begin{abstract}
A field in the vacuum state, which is in principle separable, can evolve to an entangled state in a dynamical gravitational collapse. We will study, quantify, and discuss the origin of this entanglement, showing that it could even reach the maximal entanglement limit for low frequencies or very small black holes, with consequences in micro-black hole formation and the final stages of evaporating black holes. This entanglement provides quantum information resources between the modes in the asymptotic future (thermal Hawking radiation) and those which fall to the event horizon. We will also show that fermions are more sensitive than bosons to this quantum entanglement generation. This fact could be helpful in finding experimental evidence of the genuine quantum Hawking effect in analog models.
\end{abstract}

DOI: 10.1103/PhysRevD.82.064028

PACS numbers: 04.70.Dy, 03.67.-a, 04.62.+v

\section{INTRODUCTION}

Quantum entanglement has been recognized to play a key role in black hole thermodynamics and the fate of information in the presence of horizons; some previous studies were performed in stationary cases, namely, the eternal acceleration scenario and the stationary eternal Schwarzschild black hole [1-8], not addressing issues related with dynamics and time evolution of gravitating quantum fields. On the other hand some studies involving entanglement dynamics in expanding universe scenarios have shown that the interaction with the gravitational field can produce entanglement between quantum field modes $[9,10]$.

In this paper we analyze the issue of entanglement production in a dynamical gravitational collapse. With this aim, we consider both a bosonic (scalar) and a fermionic (Grassmann scalar) field which initially are in the vacuum state and compute their asymptotic time evolution under the gravitational interaction in a stellar collapse. The vacuum state evolves to an entangled state of modes in the future null infinity (which gives rise to Hawking radiation [11]) and modes that do not reach it because they fall into the forming event horizon.

We will argue that the initial vacuum state in the asymptotic past does not have any physical quantum entanglement, and that it evolves to a state that is physically entangled as a consequence of the creation of the event horizon. This entanglement depends on the mass of the black hole and the frequency of the field modes. In particular, for very small frequencies or very small black holes, a maximally entangled state could be produced.

The entanglement generated in a gravitational collapse thus appears as a quantum resource for nondemolition methods aiming to extract information about the field modes which fall into the horizon from the outgoing Hawking radiation. These methods would be most relevant for cases such as the formation of micro-black holes and the final stages of an evaporating black hole when the mass is getting smaller and, therefore, quantum correlations generated between the Hawking radiation and the infalling modes grow to become even maximal, as we will show.

Earlier works proved that fermions and bosons have qualitatively different behaviors in phenomena such as the Unruh entanglement degradation [2,6,7] and the entanglement generation in the background of expanding universes $[9,10]$. Here, we will show that for fermions the generation of entanglement due to gravitational collapse is more robust than for bosons. This robustness is more evident from the peak of the thermal spectrum of Hawking radiation towards the ultraviolet.

Previous works in the literature (see for example [12-15] among many others) showed that Hawking radiation is correlated with the field state falling into the collapsing star. However, neither the analysis of the associated entanglement entropy as a function of the black hole parameters nor the comparison between fermionic and bosonic behavior have been carried out so far. The study of these issues, the nature of the entanglement produced in a gravitational collapse and, more important, its dependence on the nature of the quantum field (bosonic/ fermionic) is decisive in order to gain a deeper understanding about quantum entanglement in general relativistic scenarios as it was proven for other setups such as acceleration horizons, eternal black holes, and expanding universes $[2,6,7,9,10]$.

Since entanglement is a pure quantum effect, understanding its behavior in these scenarios can well be relevant to discerning the genuine quantum Hawking radiation from classical induced emission in black hole analogs [16] (see, for example, Ref. [17]), where both classical and quantum perturbations obey the same evolution laws. It will also follow from our study that fermionic modes could be more 
suitable for this task since they are more reliable in encoding entanglement information.

Finally, we will argue that the entanglement between the infalling and the Hawking radiation modes neither existed as a quantum information resource nor could have been acknowledged by any observer before the collapse occurs, namely, in the asymptotic past. This is important in order to understand the dynamics of the creation of correlations in the gravitational collapse scenario since these correlations are exclusively due to quantum entanglement, as discussed in the literature [12-15].

\section{GRAVITATIONAL COLLAPSE}

In order to analyze the entanglement production induced by gravitational collapse we will consider the Vaidya dynamical solution to Einstein equations (see e.g. Ref. [13]) that, despite its simplicity, contains all the ingredients relevant to our study. Refinements of the model to make it more realistic only introduce subleading corrections. The Vaidya space-time (Fig. 1) is conveniently described in terms of ingoing Eddington-Finkelstein coordinates by the metric

$$
\mathrm{d} s^{2}=-\left(1-\frac{2 M(v)}{r}\right) \mathrm{d} v^{2}+2 \mathrm{~d} v \mathrm{~d} r+r^{2} \mathrm{~d} \Omega^{2},
$$

where $r$ is the radial coordinate, $v$ is the ingoing null coordinate, and $M(v)=m \theta\left(v-v_{0}\right)$. For $v_{0}<v$ this is nothing but the ingoing Eddington-Finkelstein representation for the Schwarzschild metric whereas for $v<v_{0}$ it is just Minkowski space-time. This metric represents a radial ingoing collapsing shockwave of radiation. As it can be seen in Fig. 1, $v_{\mathrm{H}}=v_{0}-4 m$ represents the last null ray that can escape to the future null infinity $I^{+}$and hence that will eventually form the event horizon.

Let us now introduce two different bases of solutions to the Klein-Gordon equation in this collapsing space-time. On the one hand, we shall define the "in" Fock basis in
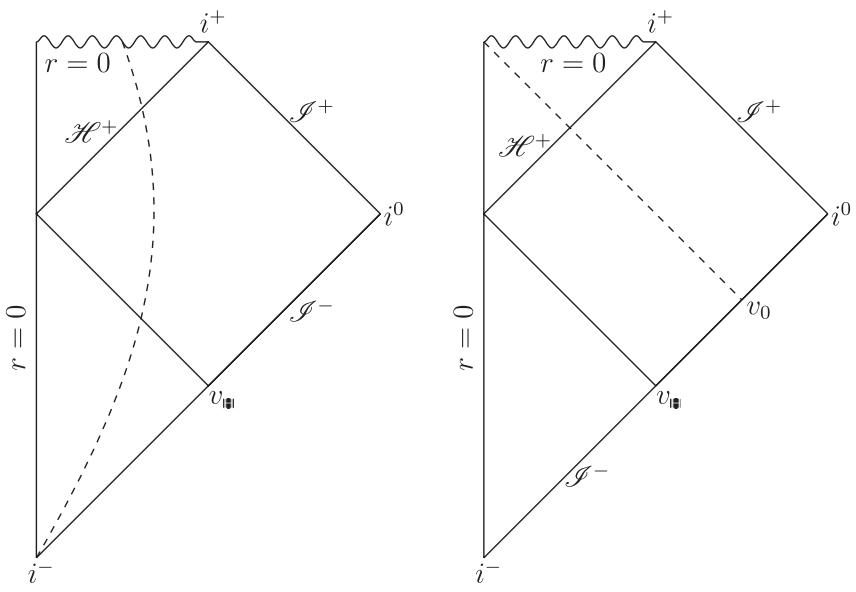

FIG. 1. Carter-Penrose diagrams for gravitational collapse: Stellar collapse (left) and Vaidya space-time (right). terms of ingoing positive frequency modes, associated with the natural time parameter $v$ at the null past infinity ${I^{-}}^{-}$, which is a Cauchy surface:

$$
u_{\omega}^{\text {in }} \sim \frac{1}{4 \pi r \sqrt{\omega}} e^{-i \omega v} .
$$

On the other hand, we can also consider an alternative Cauchy surface in the future to define another basis. In this case, the asymptotic future $I^{+}$is not enough and we also need the future event horizon $\mathcal{H}^{+}$. The "out" modes defined as being outgoing positive frequency in terms of the natural time parameter $\eta_{\text {out }}$ at $I^{+}$are

$$
u_{\omega}^{\text {out }} \sim \frac{1}{4 \pi r \sqrt{\omega}} e^{-i \omega \eta_{\text {out }}}
$$

where $\eta_{\text {out }}=v-2 r_{\text {out }}^{*}$ and $r_{\text {out }}^{*}$ is the radial tortoise coordinate in the Schwarzschild region. It can be shown (see e.g. Ref. [13]) that, for late times $\eta_{\text {out }} \rightarrow \infty$ at $I^{+}$, these modes $u_{\omega}^{\text {out }}$ are concentrated near $v_{\mathrm{H}}$ at $I^{-}$and have the following behavior:

$$
u_{\omega}^{\text {out }} \approx \frac{1}{4 \pi r \sqrt{\omega}} e^{-i \omega\left(v_{\mathrm{H}}-4 m \ln \left(\left|v_{\mathrm{H}}-v\right| / 4 m\right)\right)} \theta\left(v_{\mathrm{H}}-v\right) .
$$

These modes have only support in the region $v<v_{\mathrm{H}}$. This is evident as only the light rays that depart from $v<v_{\mathrm{H}}$ will reach the asymptotic region $\mathrm{I}^{+}$since the rest will fall into the forming horizon defined by $v=v_{\mathrm{H}}$. This is the only relevant regime, as far as entanglement production is concerned.

For the "hor" modes defined at $\mathcal{H}^{+}$, there is no such natural time parameter. A simple way to choose these modes is defining them as the modes that in the asymptotic past $I^{-}$behave in the same way as $u_{\omega}^{\text {out }}$ but defined for $v>v_{\mathrm{H}}$, that is to say, as modes that leave the asymptotic past but do not reach the asymptotic future since they will fall into the horizon. This criterion is the simplest that clearly shows the generation of quantum entanglement between the field in the horizon and the asymptotic region. In any case, since we will trace over all modes at the horizon, the choice of such modes does not affect the result. Therefore, we define the incoming modes crossing the horizon by reversing the signs of $v_{\mathrm{H}}-v$ and $\omega$ in (4) so that, near $I^{-}$, these modes are

$$
u_{\omega}^{\mathrm{hor}} \sim \frac{1}{4 \pi r \sqrt{\omega}} e^{i \omega\left(v_{\mathrm{H}}-4 m \ln \left(\left|v_{\mathrm{H}}-v\right| / 4 m\right)\right)} \theta\left(v-v_{\mathrm{H}}\right) .
$$

We are now ready to write the annihilation operators of bosonic field modes in the asymptotic past in terms of the corresponding creation and annihilation operators defined in terms of modes in the future:

$$
\begin{aligned}
a_{\omega^{\prime}}^{\text {in }}= & \int d \omega\left[\alpha_{\omega \omega^{\prime}}^{*}\left(a_{\omega}^{\text {out }}-\tanh r_{\omega} a_{\omega}^{\mathrm{hor}^{\dagger}}\right)\right. \\
& \left.+\alpha_{\omega \omega^{\prime}} e^{i \varphi}\left(a_{\omega}^{\mathrm{hor}}-\tanh r_{\omega} a_{\omega}^{\text {out }^{\dagger}}\right)\right],
\end{aligned}
$$


where $\tanh r_{\omega}=e^{-4 \pi m \omega}$. The precise values of $\varphi$ and $\alpha_{\omega \omega^{\prime}}$ are not relevant for this analysis.

Hence the vacuum $|0\rangle_{\text {in }}$, annihilated by (6) for all frequencies $\omega^{\prime}$, acquires the following form in terms of the "out-hor" basis:

$$
|0\rangle_{\text {in }}=N \exp \left(\sum_{\omega} \tanh r_{\omega} a_{\omega}^{\text {hor }^{\dagger}} a_{\omega}^{\text {out }^{\dagger}}\right)|0\rangle_{\text {hor }}|0\rangle_{\text {out }},
$$

where $N=\left(\prod_{\omega} \cosh r_{\omega}\right)^{-1}$ is a normalization constant. We can rewrite this state in terms of modes $\left|n_{\omega}\right\rangle$ with frequency $\omega$ and occupation number $n$ as

$$
|0\rangle_{\text {in }}=\prod_{\omega} \frac{1}{\cosh r_{\omega}} \sum_{n=0}^{\infty}\left(\tanh r_{\omega}\right)^{n}\left|n_{\omega}\right\rangle_{\mathrm{hor}}\left|n_{\omega}\right\rangle_{\mathrm{out}} .
$$

\section{ANALYZING ENTANGLEMENT}

This is a two-mode squeezed state. Therefore, it is a pure entangled state of the modes in the asymptotic future and the modes falling across the event horizon. Given the tensor product structure no entanglement is created between different frequency modes. Hence, we will concentrate the analysis in one single arbitrary frequency $\omega$.

We can compute the entropy of entanglement for this state which is the ultimate entanglement measure for a bipartite pure state, defined as the Von Neumann entropy of the reduced state obtained upon tracing over one of the subsystems of the bipartite state. To compute it we need the partial state $\rho_{\text {out }}=\operatorname{tr}_{\text {hor }}\left(|0\rangle_{\text {in }}\langle 0|\right)$, which turns out to be $\rho_{\text {out }}=\prod_{\omega} \rho_{\text {out }, \omega}$, where

$$
\rho_{\text {out }, \omega}=\frac{1}{\left(\cosh r_{\omega}\right)^{2}} \sum_{n=0}^{\infty}\left(\tanh r_{\omega}\right)^{2 n}\left|n_{\omega}\right\rangle_{\text {out }}\left\langle n_{\omega}\right| .
$$

This is, indeed, a thermal radiation state whose temperature is nothing but the Hawking temperature $(8 \pi m)^{-1}$, as it can be easily seen. However, this is only the partial state of the field, not the complete quantum state, which is globally entangled. If we compute the entropy of entanglement $S_{E, \omega}=\operatorname{tr}\left(\rho_{\text {out }, \omega} \log _{2} \rho_{\text {out }, \omega}\right)$ for each frequency, after some calculations, we obtain

$S_{E, \omega}=\left(\cosh r_{\omega}\right)^{2} \log _{2}\left(\cosh r_{\omega}\right)^{2}-\left(\sinh r_{\omega}\right)^{2} \log _{2}\left(\sinh r_{\omega}\right)^{2}$,

which is displayed in Fig. 2. As (8) is a pure state, all the correlations between modes at the horizon and modes in the asymptotic region are due to quantum entanglement.

Analogously we can compute the entanglement for fermionic fields. If we consider a spinless Dirac field (either one dimensional or a Grassmann scalar), the analysis is entirely analogous considering now both particle and antiparticle modes. We assume again that the initial state of the field is the vacuum that, after some long nontrivial calculations, can be expressed it in the Fock basis at the asymptotic future and the "hor" modes:

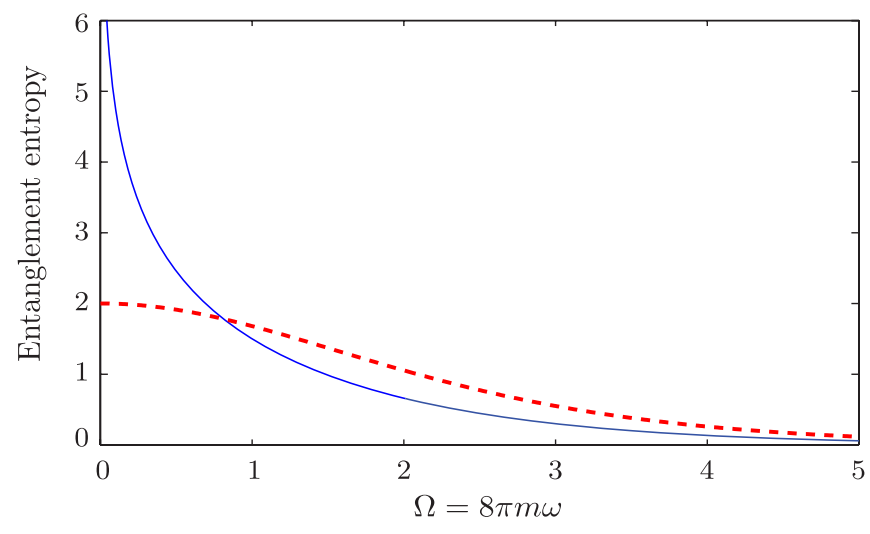

FIG. 2 (color online). Entanglement between bosonic (continuous blue line) and fermionic (red dashed line) field modes in $\mathcal{H}^{+}$and in $\mathcal{I}^{+}$. The lesser the mass of the star or the mode frequency, the higher the entanglement reached.

$$
\begin{aligned}
|0\rangle_{\text {in }}= & \prod_{\omega}\left[\left(\cos \tilde{r}_{\omega}\right)^{2}|00\rangle_{\text {hor }}|00\rangle_{\text {out }}-\frac{\sin 2 \tilde{r}_{\omega}}{2}\right. \\
& \times\left(\left|01_{\omega}\right\rangle_{\text {hor }}\left|1_{\omega} 0\right\rangle_{\text {out }}-\left|1_{\omega} 0\right\rangle_{\text {hor }}\left|01_{\omega}\right\rangle_{\text {out }}\right) \\
& \left.-\left(\sin \tilde{r}_{\omega}\right)^{2}\left|1_{\omega} 1_{\omega}\right\rangle_{\text {hor }}\left|1_{\omega} 1_{\omega}\right\rangle_{\text {out }}\right],
\end{aligned}
$$

where $\tan \tilde{r}_{\omega}=e^{-4 \pi m \omega}$. Here, we are using the double Fock basis, the first figure inside each bracket representing particles and the second antiparticles.

We can compute the entropy of entanglement of this pure state. The partial density matrix in the asymptotic future $\rho_{\text {out }}=\operatorname{tr}_{\text {hor }}\left(|0\rangle_{\text {in }}\langle 0|\right)=\prod_{\omega} \rho_{\text {out }, \omega}$ is given by

$$
\begin{aligned}
\rho_{\text {out }, \omega}= & \left(\cos \tilde{r}_{\omega}\right)^{4}|00\rangle_{\text {out }}\langle 00|+\frac{\left(\sin 2 \tilde{r}_{\omega}\right)^{2}}{4}\left(\left|1_{\omega} 0\right\rangle_{\text {out }}\left\langle 1_{\omega} 0\right|\right. \\
& \left.+\left|01_{\omega}\right\rangle_{\text {out }}\left\langle 01_{\omega}\right|\right)+\left(\sin \tilde{r}_{\omega}\right)^{4}\left|1_{\omega} 1_{\omega}\right\rangle_{\text {out }}\left\langle 1_{\omega} 1_{\omega}\right|,
\end{aligned}
$$

which is again a thermal state with Hawking temperature $(8 \pi m)^{-1}$, and

$$
S_{E, \omega}=-2\left[\left(\cos \tilde{r}_{\omega}\right)^{2} \log _{2}\left(\cos \tilde{r}_{\omega}\right)^{2}+\left(\sin \tilde{r}_{\omega}\right)^{2} \log _{2}\left(\sin \tilde{r}_{\omega}\right)^{2}\right]
$$

which is also displayed in Fig. 2.

Figure 2 shows that the entanglement decreases as the mass of the black hole or the frequency of the mode increase. When comparing bosons with fermions one must have in mind that the entropy of entanglement is bounded by (the logarithm of) the dimension of the partial Hilbert space ("out" Fock space in our case). Therefore, due to the Pauli exclusion principle, the maximum entropy of entanglement for fermions is $S_{E, \omega}=2$, which corresponds to a maximally entangled state. On the other hand, for bosons, the entanglement is distributed among the superposition of all the occupation numbers and the entropy can grow unboundedly, reaching the maximally entangled state in the limit of infinite entropy. In this sense, 
the entanglement generated in the fermionic case is more useful and robust due to the limited dimension of the Fock space for each fermionic mode.

This result can be traced back to the inherent differences between fermions and bosons. Specifically, it is the Pauli exclusion principle which makes fermionic entanglement more reliable. Similar results about reliability of entanglement for fermions were also found in the expanding universe scenarios [10]. This responds to the high influence of statistics in entanglement behavior in general relativistic settings as it was investigated in $[6,7]$. On the other hand, Vaidya space-time has all the fundamental features of a stellar collapse and shows how the entanglement is created by the appearance of an event horizon. Hence, in other collapsing scenarios or including the subleading grey-body factor corrections, these fundamental statistical differences will not disappear. The qualitatively different behavior of entanglement for bosons and fermions is not an artifact of choosing a particular collapse scenario but is due to fundamental statistical principles.

In the above analysis we have considered plane wave modes, which are completely delocalized. However, an entirely analogous analysis can be easily carried out using very well localized Gaussian states, with the same results about quantum entanglement behavior.

\section{CONCLUSIONS}

We have shown that the formation of an event horizon generates entanglement. If we start from the vacuum state in the asymptotic past, after the gravitational collapse process is complete we end up with a state in the asymptotic future which shares pure quantum correlations with the field modes which fall into the horizon. One could think that this entanglement was already present before the collapse, arguing that (as proved in [18]) the vacuum state of a quantum field can be understood as an entangled state of spacelike separated regions. In other words, if we artificially divided the Cauchy surface in which the vacuum state is determined into two parts, we would have a quantum correlated state between the two partitions. In principle we could have done a bipartition of the vacuum state in $I^{-}$such that it would reflect entanglement between the partial state of the vacuum for $v<v_{\mathrm{H}}$ and the corresponding partial state for $v>v_{\mathrm{H}}$. However, it is not until the collapse occurs that we have the information about what $v_{\mathrm{H}}$ is. So, achieving beforehand the right bipartition (trying to argue that the entanglement was already in the vacuum state) would require a complete knowledge of the whole future and, consequently, there is no reason a priori to do such bipartition. The entanglement, eventually generated by the collapse, will remain unnoticed to early observers, who are deprived of any means to acknowledge and use it for quantum information tasks. It is well known that if we introduce artificial bipartitions of a quantum system, its description can show entanglement as a consequence of the partition. However, not being associated with a physical bipartition this entanglement does not codify any physical information and, hence, cannot be used to perform any quantum information processes. (One example of this kind of nonuseful entanglement is statistical entanglement between two undistinguishable fermions [19]).

Gravitational collapse selects a specific partition of the initial vacuum state by means of the creation of an event horizon. In the asymptotic past there was no reason to consider a specific bipartition of the vacuum state, whereas in the future there is a clear physically meaningful bipartition: What in $I^{-}$was expressed as a separable state, now becomes expressed in terms of modes that correspond to the future null infinity and the ones which fall across the event horizon. This means that gravitational collapse defines a particular physical way to break the arbitrariness of bipartitioning the vacuum into different subsystems. This gravitational production of entanglement would be a physical realization of the potentiality of the vacuum state to be an entangled state and is therefore a genuine entanglement creation process.

We have computed the explicit functional form of this entanglement and its dependence on the mass of the black hole (which determines the surface gravity). For more complicated scenarios (with charge or angular momentum), it will depend on these parameters as well.

For small black holes, the outgoing Hawking radiation tends to be maximally entangled with the state of the field falling into the horizon for both bosons and fermions. This means that if a hypothetical high energy process generates a micro-black hole, a projective measurement carried out on the emitted radiation (as, for instance, the detection of Hawking radiation) will "collapse" the quantum state of the field that is falling into the event horizon and give us certainty about the outcome of possible measurements carried out in the vicinity of the horizon. Furthermore, at least theoretically speaking, the available quantum information resources would be maximum and, therefore, one could perform quantum information tasks such as quantum teleportation with maximum fidelity from the infalling modes to the modes in the asymptotic future $\mathrm{I}^{+}$if the observer of the infalling modes managed to dispatch an outgoing classical signal before crossing the horizon. On the other hand, low frequency modes become more entangled than the higher ones. So, the infrared part of the Hawking spectrum would provide more information about the state at the horizon than the ultraviolet.

Arguably, similar conclusions can be drawn for the final stages of an evaporating black hole: As the mass of the black hole diminishes, the temperature of the Hawking radiation spectrum increases, and therefore, the quantum state of the field tends to a maximally entangled one in the limit of $m \rightarrow 0$.

We have seen that the entanglement generated in fermionic fields is more robust than for bosons. Although the 
entropy of entanglement in the zero mass limit is greater in the bosonic case due to the higher dimension of the partial Hilbert space, we have argued that the information is more reliably encoded in the limited Fock space of fermionic fields. Furthermore, as we consider higher frequency modes, fermionic entanglement proves to be much more easily created by the collapse. What is more, the turning point in which the entropy of entanglement for fermions becomes numerically larger than for bosons is actually near the peak of the thermal emission (Fig. 2). This means, that, in general, a measurement carried out on Hawking radiation of fermionic particles will give us more information about the near-horizon field state. This might also be useful in analog gravity realizations as we have already discussed, specifically in systems where the field excitations are fermionic (see e.g. Ref. [20]), which would be, as shown, at an advantage over the bosonic cases. To account for this quantum entanglement in analog experiments one should carry out measurements of the quantum correlations between the emitted thermal spectrum and the infalling modes and detect Bell inequalities violations. This is easier as it gets closer to the maximally entangled case.

\section{ACKNOWLEDGMENTS}

The authors want to thank Carlos Barceló for useful discussions. This work was supported by the Spanish MICINN Projects FIS2008-05705/FIS, FIS2008-06078C03-03, the CAM research consortium QUITEMAD S2009/ESP-1594, and the Consolider-Ingenio 2010 Program CPAN (CSD2007-00042). E. M-M. was partially supported by a CSIC JAE-PREDOC2007 grant.
[1] P.M. Alsing and G.J. Milburn, Phys. Rev. Lett. 91, 180404 (2003).

[2] I. Fuentes-Schuller and R. B. Mann, Phys. Rev. Lett. 95, 120404 (2005).

[3] P.M. Alsing, I. Fuentes-Schuller, R. B. Mann, and T.E. Tessier, Phys. Rev. A 74, 032326 (2006).

[4] J. León and E. Martín-Martínez, Phys. Rev. A 80, 012314 (2009).

[5] E. Martín-Martínez and J. León, Phys. Rev. A 80, 042318 (2009).

[6] E. Martín-Martínez and J. León, Phys. Rev. A 81, 032320 (2010).

[7] E. Martín-Martínez and J. León, Phys. Rev. A 81, 052305 (2010).

[8] E. Martín-Martínez, L. J. Garay, and J. León, Phys. Rev. D 82, 064006 (2010)

[9] J.L. Ball, I. Fuentes-Schuller, and F. P. Schuller, Phys. Lett. A 359, 550 (2006).
[10] I. Fuentes, R. B. Mann, E. Martín-Martínez, and S. Moradi, Phys. Rev. D 82, 045030 (2010).

[11] S. W. Hawking, Nature (London) 248, 30 (1974).

[12] R. Balbinot, A. Fabbri, S. Fagnocchi, A. Recati, and I. Carusotto, Phys. Rev. A 78, 021603 (2008).

[13] A. Fabbri and J. Navarro-Salas, Modeling Black Hole Evaporation (World Scientific, Singapore, 2005).

[14] R. Schützhold and W. G. Unruh, Phys. Rev. D 81, 124033 (2010).

[15] S. Fagnocchi, J. Phys. Conf. Ser. 222, 012036 (2010).

[16] W. G. Unruh, Phys. Rev. Lett. 46, 1351 (1981).

[17] B. Horstmann, B. Reznik, S. Fagnocchi, and J. I. Cirac, Phys. Rev. Lett. 104, 250403 (2010).

[18] B. Reznik, A. Retzker, and J. Silman, Phys. Rev. A 71, 042104 (2005).

[19] J. Schliemann, J. I. Cirac, M. Kuś, M. Lewenstein, and D. Loss, Phys. Rev. A 64, 022303 (2001).

[20] G. E. Volovik, The Universe in a Helium Droplet (Oxford University Press, New York, 2003). 